\title{
Optimization-based worst-case analysis of a launcher during the atmospheric ascent phase
}

\author{
Andrés Marcos ${ }^{1}$ and Hector García de Marina ${ }^{2}$ \\ Deimos Space SLU, Madrid, Spain, 28035 \\ Valerio Mantini ${ }^{3}$, Christophe Roux ${ }^{4}$ \\ ELV, Corso Garibaldi 22, 00034, Colleferro (Rome), Italy \\ Samir Bennani ${ }^{5}$ \\ ESA-ESTEC, Keplerlaan 1, 2201AZ, Noordwijk, The Netherlands
}

\begin{abstract}
This article presents the application of optimization-based worst-case search approaches to the VEGA launcher during the P80 ascent phase. Four optimization algorithms are applied covering evolutionary and deterministic global methods as well as their hybrid versions, i.e. mixed with local approaches. A comparison with a traditional Monte Carlo campaign shows that the optimization-based algorithms are much amenable to worst-case search.
\end{abstract}

\section{Introduction}

$\mathrm{T}$ HE current state-of-practice for launcher Verification \& Validation (V\&V) entails analysis of frequency domain requirements through a set of predefined vertex cases and more critically, analysis of time domain requirements through nonlinear simulation Monte Carlo campaigns (i.e. probabilistic approach) and using a set of selected worstcases (i.e. deterministic approach).

Traditional MC campaigns consist of randomly sampling the uncertain parameters according to statistical distributions and deducing the values of criteria involved in the requirements. The number of simulations depends on the specified requirements probability and confidence levels. Although the MC approach is very practical in showing the design sensitiveness to parametric variations, it has many disadvantages in that it relies on massive amounts of computations without guaranteed proofs on the full parameter space and is questionable for maximum/minimum values analysis.

Similarly, vertex approaches, whereby all the corner cases (min,max) are checked, are unfeasible whenever the dimension of the problem is relatively high. This is compensated by introducing engineering knowledge on the system which helps limit the number of corner cases to be examined. Nonetheless, the analysis is very limited and obviate altogether parameter combinations away from the extremal values.

To overcome these limitations many advances have been explored in the field of aeronautical and military GNC $\mathrm{V} \& \mathrm{~V}$ based on advanced optimization-based worst-case search algorithms and tools ${ }^{1,2,3}$. Some of these algorithms use the gradient information of the cost function to find the search direction while others use only the cost function value. Many of these non-gradient based techniques make use of heuristic search directions in an efficient and intelligent way to mix local and global properties. Furthermore, depending on the form an algorithm optimizes its search direction the result might be a global or a local optimum. Obviously, if the search space is convex, in

${ }^{1}$ RFCS project manager, Control Systems Center of Competence, Deimos Space, Senior AIAA member.

${ }^{2}$ Now PhD candidate at the University of Groningen, The Netherlands.

${ }^{3}$ Consultant, GNC department, Altran.

${ }^{4}$ VEGA Control leader, GNC department, ELV.

${ }^{5}$ ESA expert, Guidance Navigation \& Control System Department, ESA/ESTEC, Senior AIAA member. 
principle, both local and global optimization algorithms will converge to the true global solution. In the case of a non-convex search space, gradient-based optimization algorithms provide a local solution, rather than the true global solution. The performance of a given optimization algorithm is generally problem dependent, and there is no unique optimization algorithm for general classes of problems which will guarantee computation of the true global solution with reasonable computational complexity.

A European Space Agency (ESA) study led by Deimos and entitled "Robust Flight Control System Design Verification and Validation Framework" (RFCS) ${ }^{4}$ was established with the objective of developing, demonstrating and comparing with a traditional $\mathrm{V} \& \mathrm{~V}$ framework a new enhanced design $\mathrm{V} \& \mathrm{~V}$ framework through their application to the V\&V of a complex launch vehicle. The selected launcher was VEGA, the new European Small Launch Vehicle (LV) developed under the responsibility of ESA by ELV as the prime contractor. A successful maiden flight of VEGA was performed on the $13^{\text {th }}$ February 2012 with a $2^{\text {nd }}$ flight on the $7^{\text {th }}$ May 2013.

Within RFCS, a number of different optimization algorithms were applied to assess the robust performance of the VEGA during the P80 flight phase. This article presents the results of such study. The layout is as follows: Section II introduced the VEGA launcher, associated nonlinear simulators used in the RFCS project and the worstcase problem definition. Section III shows the results from applying a traditional Monte Carlo campaign. Section IV describes the optimization-based methodology and Section V presents a selected number of results from its application and Section VI the validation between VEGACONTROL and VEGAMATH. Finally, the paper ends with tehe conclusions in Section VII.

\section{System and problem description}

\section{A. VEGA launcher}

VEGA is the new European Small Launch Vehicle developed under the responsibility of ESA. The prime contractor for the launch vehicle is ELV. The launcher enters the Qualification phase and successfully performed the first launch on the $13^{\text {th }}$ February 2012 from the Centre Spatial Guyanais in Kourou with a $2^{\text {nd }}$ flight on the $7^{\text {th }}$ May 2013.

The propulsion system of VEGA is composed of: three solid propellant motors (P80, Zefiro 23 and Zefiro 9) providing thrust for the 1st, 2nd and 3rd stages; and, a bi-propellant liquid engine (LPS) on the 4th stage (AVUM). The LPS can be re-ignited and performs up to 5 boosts. It is used for: transfer orbit injection (1st boost), scattering compensation, final orbit injection (2nd boost) and AVUM de-orbiting (last boost).

All four stages are controlled via a thrust vectoring system (TVC). The 4th stage also includes a Roll and Attitude Control System (RACS). During the propelled phase, the RACS system controls the roll rate while in ballistic phases it performs a three axes control. A strong constraint on the missions is the safety: the separated stages must fall on sea areas.

In the reference mission of VEGA, see Figure 1, a $1500 \mathrm{Kg}$ payload will be released at $700 \mathrm{Km}$ altitude on a circular Polar Earth Orbit (PEO). In the Qualification flight, a $700 \mathrm{Kg}$ payload will be released at $1450 \mathrm{Km}$ altitude circular orbit of $71 \mathrm{deg}$ inclination. Since the payload (PL) masses range from $300 \mathrm{~kg}$ up to $2500 \mathrm{~kg}$, the type of missions are manifold: equatorial, polar, SSO, multi-PL, barbeque mode in long ballistic phase and payload release with spin among others. Along the nominal trajectory, the flight parameters are subject to important - though predictable - variations versus time.
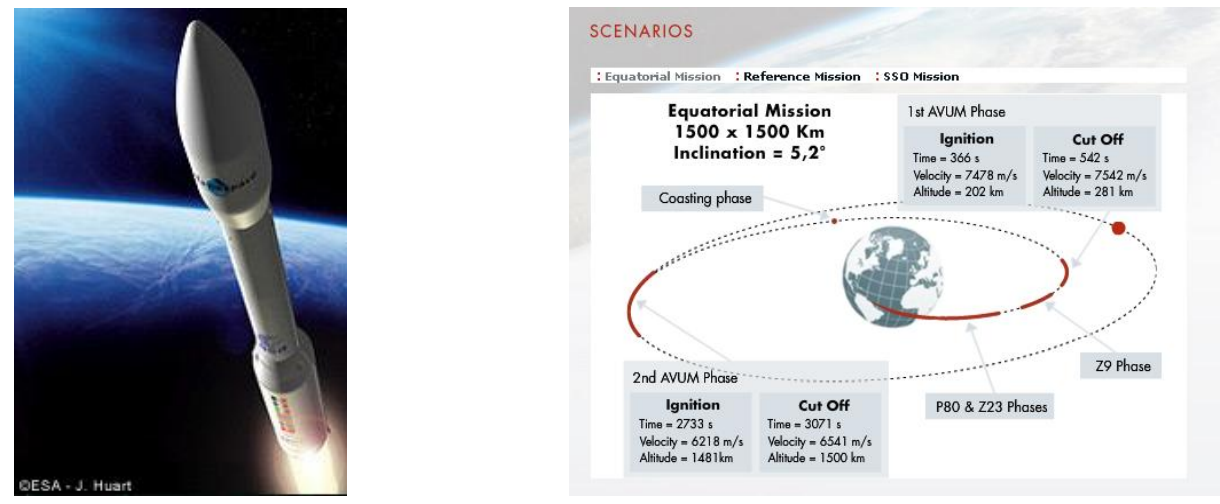

Figure 1 VEGA launcher and mission scenarios

2

American Institute of Aeronautics and Astronautics 


\section{B. VEGA nonlinear simulators}

The VEGA nonlinear simulator used in the RFCS project is the nonlinear 6 degrees-of-freedom model called VEGACONTROL, which simulates in the time-domain the VEGA flight during the P80 phase. The simulator, see Figure 2, is prepared to perform the LARES or DT1 missions based on a $700 \mathrm{Kg}$ payload (PL) and with the following specific characteristics:

- No bending or sloshing modes,

- No RACS model,

- Detailed launcher vehicle model, including high-fidelity Aerodynamic database

- TVC system, including computing delays

- Guidance, navigation and control (GNC) system

- Propulsion and mass-center-inertia (MCI) properties

- Inertial navigation system (INS),

VEGACONTROL is used by the RFCS consortium to develop and verify their approaches, and the results from these verifications are validated (by ELV) on an equivalent simulator called VEGAMATH. This $2^{\text {nd }}$ simulator is the official VEGA program nonlinear, time-domain $6 \mathrm{DoF}$ simulator.
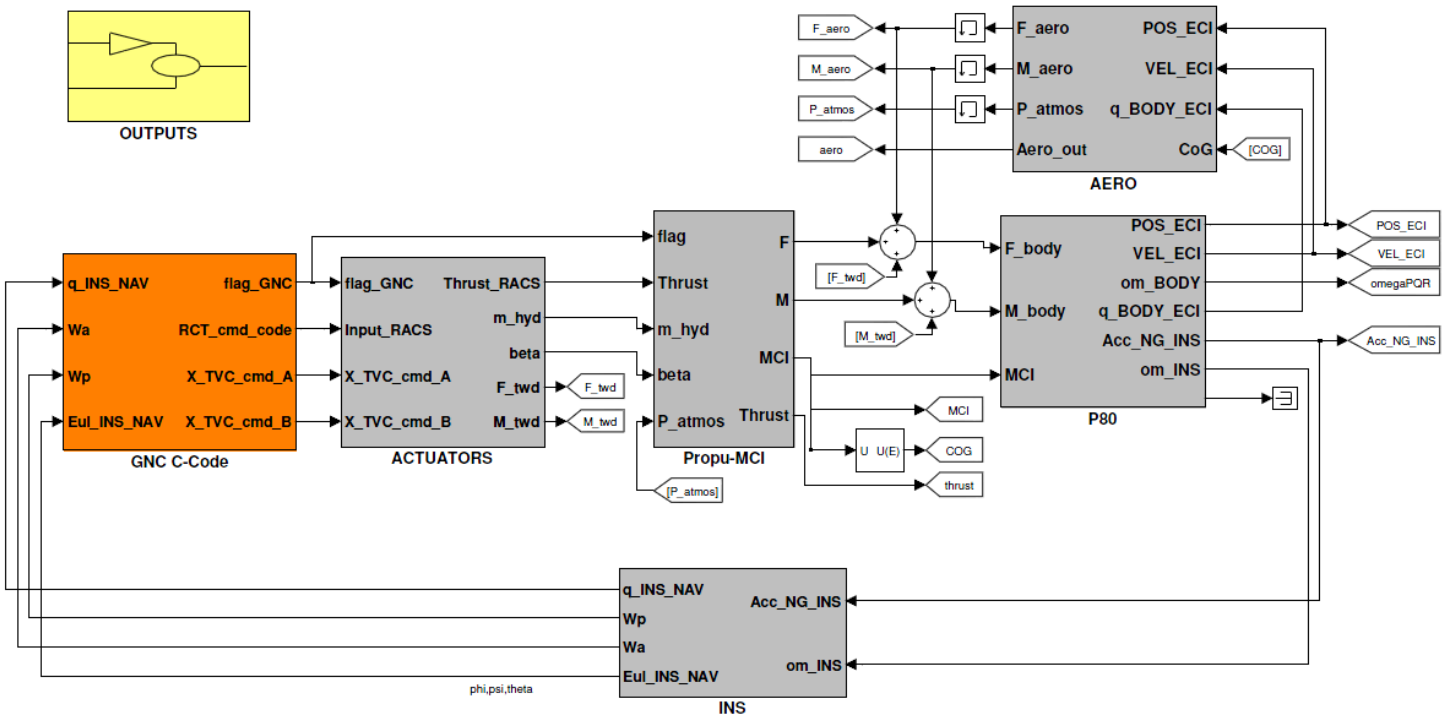

Figure 2 VEGACONTROL Simulink block implementation

Both simulators allow modifying the scattering values (uncertainties and dispersions) of up to 95 different LV uncertain parameters. Each scattering variable is multiplied by a "flag" variable included in the interval $[-1,1]$ with the zero flag value indicating "no scattering". A subset of relevant parameters can be organized into five uncertain sets depending on their nature (e.g. mass uncertainty, aerodynamic coefficient dispersion, TVC bias and misalignments): MCI, Thrust, Aerodynamics, Atmosphere and TVC.

\section{Problem description}

In order to identify worst-cases using an optimization-based procedure, a mathematical optimization problem is established. In order to do this, an objective function (i.e. performance metric) $f_{o}: R^{n} \rightarrow R$ is defined dependent on optimization variables $x=\left(x_{1}, \ldots, x_{n}\right)$ and subject to constraint inequalities $f_{i}: R^{n} \rightarrow R, i=1, \ldots, m$ and constant limits $b_{1}, \ldots, b_{m}$. In the worst- case search problem, this can be posed as finding the vector $x$ that optimizes the performance metric $f_{o}$ subject to the inequality constraints on $x$ :

\section{minimize $f_{o}(x)$}

subject to $b_{i-\min } \leq x_{i} \leq b_{i-\operatorname{maz}}$

Four objective functions are considered in this study due to their relevancy for launchers:

1. Aerodynamic Load: $\mathrm{C}_{1}(\mathrm{p})=\max (\mathrm{Q} \alpha($ Mach, $\mathrm{p}) / \mathrm{Q} \alpha$ (Mach)bound )

2. Roll Rate: $\mathrm{C}_{2}(\mathrm{p})=\max ($ roll-rate(time)/ roll-rate(time)bound )

3. Angular velocity module multiplied by the Angle of Attack: $\mathrm{C}_{3}(\mathrm{p})=|\Omega| \alpha$

4. Angular acceleration: $\mathrm{C}_{4}(\mathrm{p})=\Omega \operatorname{dot} / \Omega \operatorname{dot}$-bound

American Institute of Aeronautics and Astronautics 


\section{Traditional Monte Carlo Campaign}

Traditional Monte Carlo campaigns consist of randomly sampling the uncertain parameters according to statistical distributions and to deduce the values of criteria involved in the requirements. The number of simulations depends on the specified requirements probability and confidence levels: for example, for launcher consumption is required at least 1000 runs (99.9\% probability at $60 \%$ of confidence) while for control accuracy is sufficient with 100 runs (99\% probability and $60 \%$ confidence).

It may happen that the dependence of the criteria versus the parameters is not simple or monotonous. In this case, the statistical approach allows exploring the domain of variations. Additionally, when the requirements are specified with a level of probability and confidence, their fulfillment must be assessed in terms of probability analysis and this approach is the most natural. The main advantages of the method are its simplicity of implementation and that is useful for mean / variance analysis. The drawbacks are that it is computationally heavy and questionable for maximum/minimum values analysis or even for finding interior worst-cases. Indeed, these shortcomings will be exemplified in this section.

A traditional Monte Carlo campaign is performed using one hundred thousand $(100,000)$ random combinations of the global uncertain set (i.e. the full 95 uncertain parameters) within the range $[-1,1]$. Only 11 cases result in a cost-function value for the $\mathrm{C}_{1}>0.9$ (maximum aerodynamic load), 140 for $\mathrm{C}_{4}>0.4$ (maximum angular acceleration) and 1341 for $\mathrm{C}_{2}>0.5$ (maximum roll rate). Moreover, none of these cases violate the bound (i.e. no cost was higher than 1). Figure 3 shows the 11 cases for $C_{1}$ on the left and the 1341 for $C_{2}$ on the right.
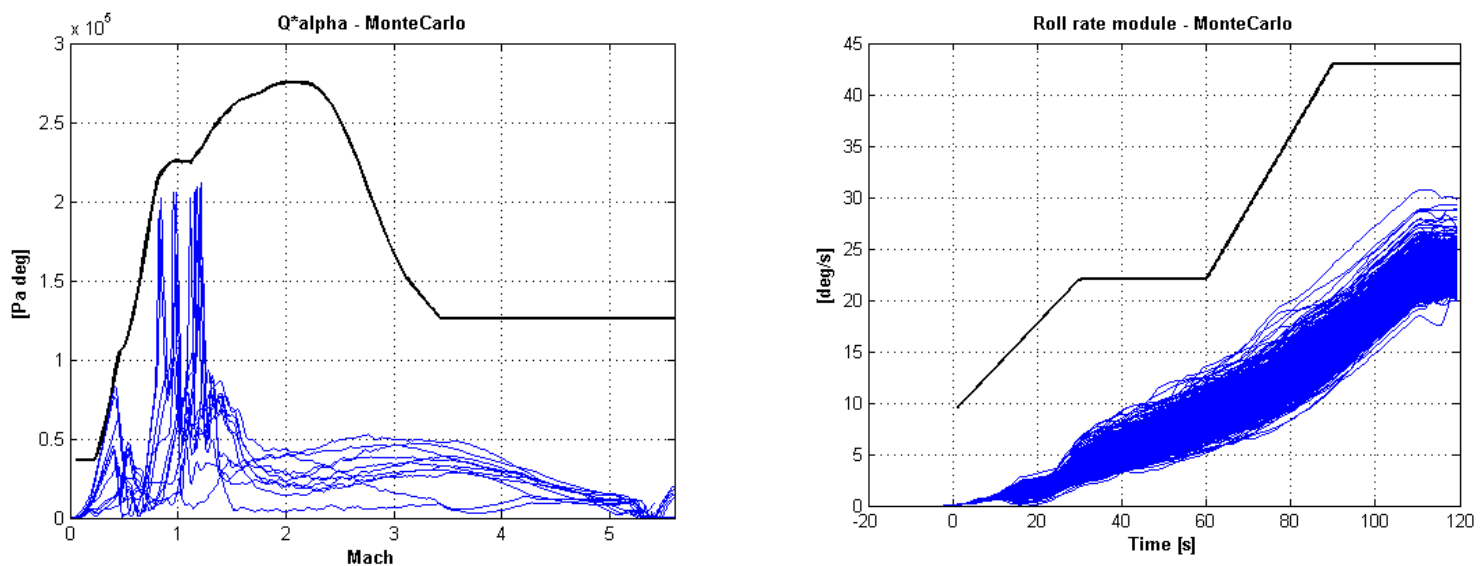

Figure 3 Traditional MC, left for $\mathrm{C} 1>0.9$ (11 cases) $\&$ right for $\mathrm{C} 2>0.5$ (1341 cases)

It is also interesting to obtain the associated probability distribution functions (PDF). In the case of $\mathrm{C}_{1}$ it is seen that all the cases simulated are within the stable region $(<1.0)$ and most around 0.3 , see Figure 4.

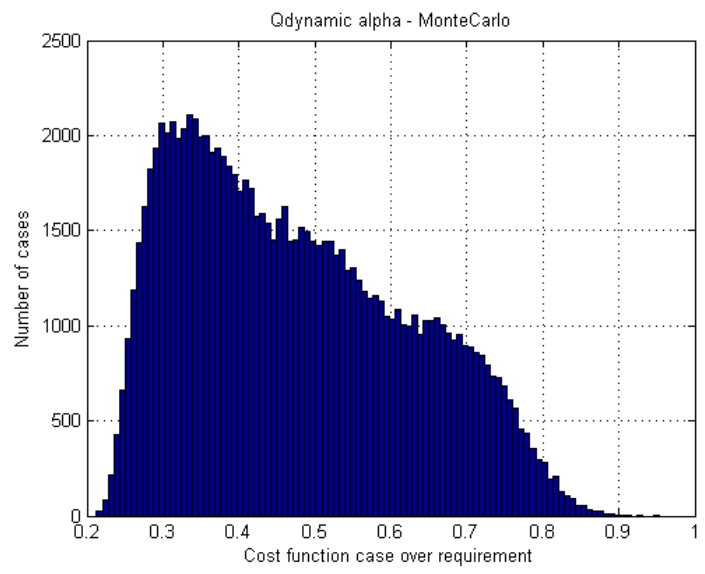

Figure 4 PDF of $\mathrm{C}_{1}$ for a $\mathrm{MC}$ campaign with 100,000 simulations 


\section{Optimization-Based Worst-Case Methodology}

Many different classes of optimization algorithms are available in the literature ${ }^{5 \rightarrow 12}$. Some of these algorithms use the gradient information of the cost function to find the search direction while others use only the cost function value. Many of these non-gradient based techniques make use of heuristic search directions in an efficient and intelligent way to mix local and global properties (the so-called hybrid approaches).

Depending on the manner an algorithm optimizes its search direction the result might be a global or a local optimum. Obviously, if the search space is convex, in principle, both local and global optimization algorithms will converge to the true global solution. In the case of a non-convex search space, gradient-based optimization algorithms provide a local solution, rather than the true global solution. The performance of a given optimization algorithm is generally problem dependent, and there is no unique optimization algorithm for general classes of problems which will guarantee computation of the true global solution with reasonable computational complexity.

Therefore, a number of different optimization algorithms are applied for the specific problem at hand. This will enable to evaluate and compare them in order that the most promising method is identified for the considered LV problem. Specifically, the following four algorithms are used (the last two are hybrid versions of the first two):

1. Differential Evolution (DE)

2. Dividing Rectangle (DIRECT)

3. Hybrid Differential Evolution (HDE)

4. Hybrid Dividing Rectangles (HDIRECT)

These algorithms are implemented in the Worst-Case Analysis Toolbox (WCAT) from Leicester University ${ }^{13}$. This toolbox was consolidated in another ESA Technology Research Project led by Deimos and entitled "Linear Parameter Varying Modeling, Analysis and Design (LPVMAD) ${ }^{14,}$.

The methodology consists mainly in applying the four optimization algorithms to the four cost functions, for each of the five uncertain sets (MIC, Thrust, Aerodynamics, Atmosphere, TVC). In order to provide a wide coverage of conditions without increasing the computational load, each of these five uncertainty set is alternatively used as the optimization vector $x$ from Eq. (1) while all the other uncertain parameters are set to the minimum/nominal/maximum values, i.e. $\{-1.0,0.0,+1.0\}$-that is there are 3 worst-case searches per uncertainty set. Further, each algorithm is applied twice varying the allowed number of iterations (i.e. first a thousand iterations are allowed and then up to five thousand). This will allow obtaining information on the performance of the algorithms by measuring the quality of the results in terms of number of iterations. Table 1 shows the worst-case condition (WCC) search tests performed by cost function, search algorithm and number of iterations.

Table 1 Worst-case condition (WCC) search tests

\begin{tabular}{|l|l|l|}
\hline Cost function & $\mathbf{1 0 0 0}$ iterations & $\mathbf{5 0 0 0}$ iterations \\
\hline $\mathbf{C}_{\mathbf{1}}(\mathbf{p})=\mathbf{m a x}\left(\mathbf{Q} \boldsymbol{\alpha}(\mathbf{M a c h}, \mathbf{p}) / \mathbf{Q} \boldsymbol{\alpha}(\mathbf{M a c h})_{\text {bound }}\right)$ & DE/HDE/DIRECT/HDIRECT & DE/HDE/DIRECT/HDIRECT \\
\hline $\left.\mathbf{C}_{\mathbf{2}}(\mathbf{p})=\mathbf{m a x}(\text { roll-rate(time)/ roll-rate(time })_{\text {bound }}\right)$ & DE/HDE & -- \\
\hline $\mathbf{C}_{\mathbf{3}}(\mathbf{p})=|\boldsymbol{\Omega}| \boldsymbol{\alpha}$ & $\mathrm{DE} / \mathrm{HDE} / \mathrm{DIRECT} / \mathrm{HDIRECT}$ & $\mathrm{DE} / \mathrm{HDE} / \mathrm{DIRECT} / \mathrm{HDIRECT}$ \\
\hline $\mathbf{C}_{\mathbf{4}}(\mathbf{p})=\boldsymbol{\Omega}_{\text {dot }} / \boldsymbol{\Omega}_{\text {dot-bound }}$ & DE/HDE/DIRECT/HDIRECT & DE/HDE/DIRECT/HDIRECT \\
\hline
\end{tabular}

Notice, that by searching for worst-cases in a set-by-set approach, a sensitivity analysis of the most critical groups of parameters is carried out. This allows to identify critical parameters that deserve more attention -for example, uncertain parameters to be used for subsequent LFT modeling towards analytical worst-case analysis.

Finally, a WCC search with all the 95 uncertainty parameters as random was performed using the $\mathrm{C}_{1}$ cost function and the four algorithms for 1000 iterations. Thus, in total, there were $394 \mathrm{WCC}$ campaigns corresponding to: the 26 test campaigns in Table 1 times 5 uncertainty sets, times 3 flag settings for the remaining uncertain parameters plus the 4 all-flags-free campaigns (i.e. the four WCCs tests using the 95 parameters).

\section{Results}

In this section, a selected summary of results is presented by focusing on the Aerodynamic and MCI uncertainty sets as well as the all-flags-free campaign.

\section{A. Aerodynamic set}

The Aerodynamic uncertain set is formed by dispersions (the scattering, or distribution, around the most likely values of the parameter) and uncertainties (the range of values of the parameter) on the following parameters: axial 
aerocoefficient \{'disp $\mathrm{CA}^{\prime}$ ', 'unc $\mathrm{CA}$ '\}, normal aerocoefficient $\left\{\right.$ 'disp $\mathrm{CN}^{\prime}$, 'unc $\left.\mathrm{CN}^{\prime}\right\}$, and $\mathrm{x}$-coordinate of pressure center $\{$ 'disp Xcp', 'unc Xcp'\}.

Figure 5 shows a WCCs violating the $\mathrm{C}_{1}$ cost function (i.e. maximal $\mathrm{Q} \alpha$ ) for each of the four algorithms and using 1000 iterations. Note that the WCCs found are interior cases (i.e. away from the minimum/maximum vertex combinations). Additionally, observe that for the DIRECT and HDIRECT algorithms the WCCs are the same. This has to do with the manner these algorithms are set-up: they perform first a pseudo-vertex approach by dividing the parameter space in rectangles and then checking each rectangle with the hybridization, which results in different WCCs if the number of iterations is large or the number of uncertain parameters small.
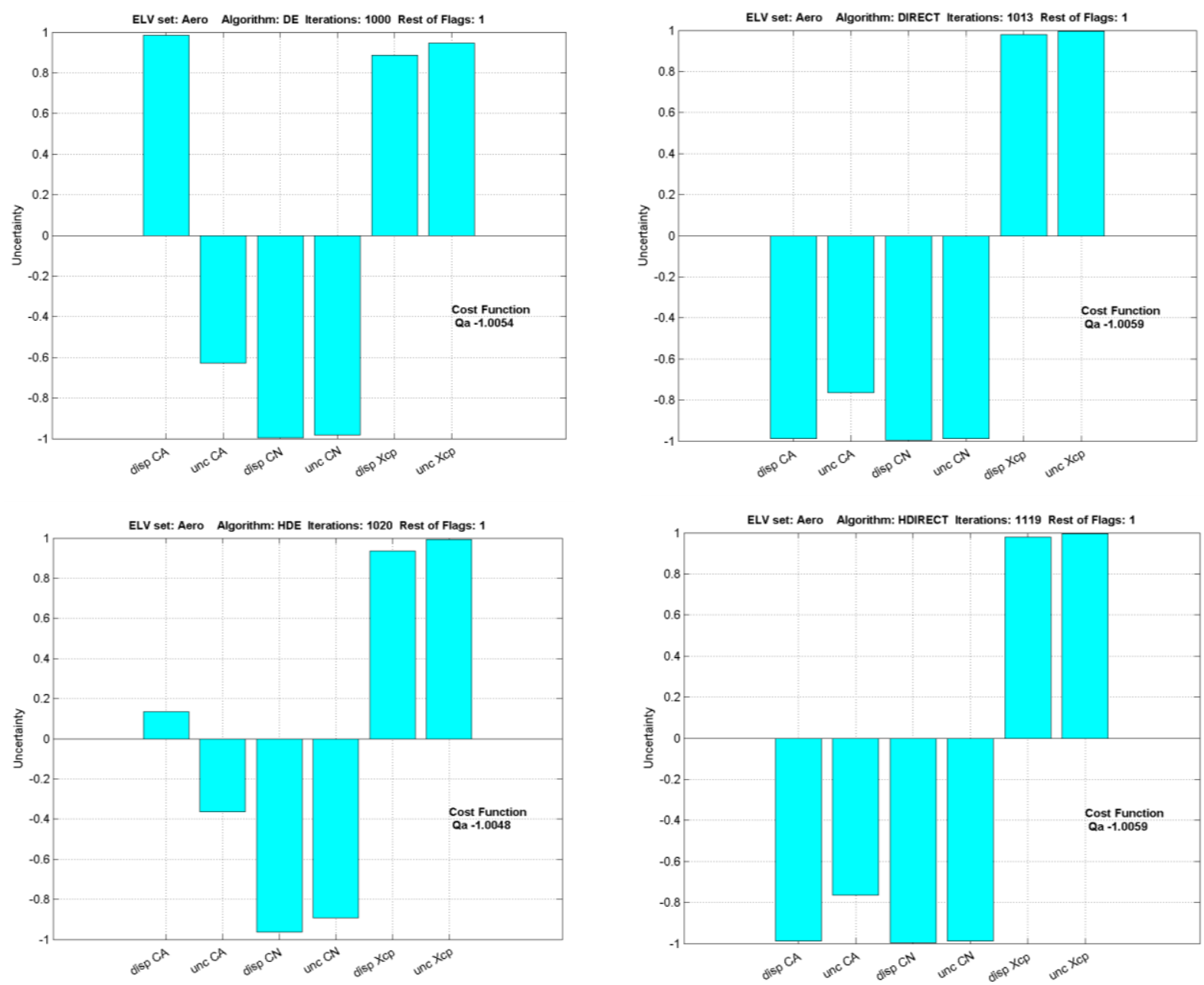

Figure 5 Aerodynamics set and $1 \mathrm{~K}$ iterations, all algorithms for $\mathrm{C}_{1}$ : WCCs

Figure 6 shows the $\mathrm{C}_{1}$ time-response corresponding to the HDE algorithm. It can be seen that the slight criteria violation (i.e. $\left|\mathrm{C}_{1}\right|=1.0048$ ) shows around Mach 4.0. 


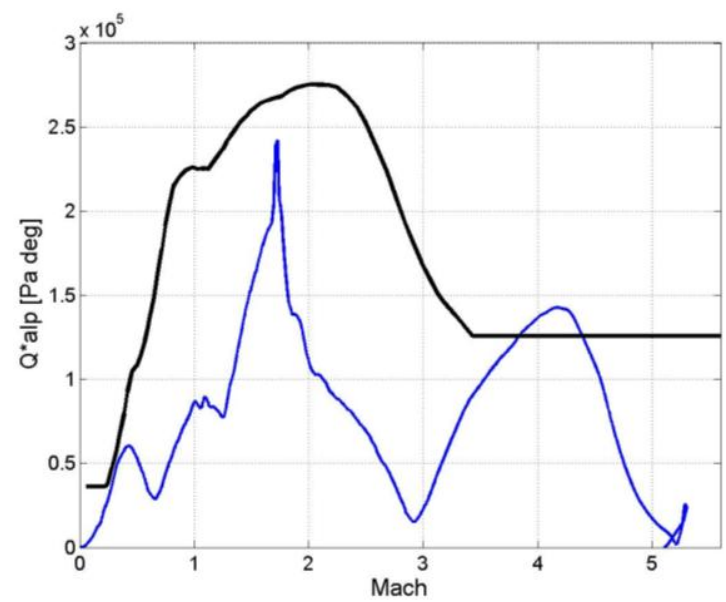

Figure 6 Aerodynamics set and $1 \mathrm{~K}$ iterations, $\mathrm{HDE}$ for $\mathrm{C}_{1}$ : time-response

Figure 7 shows the same cost function $\mathrm{C}_{1}$ but for 5000 iterations. Notice that the four algorithms start to converge to a similar WCCs with only slightly different values for \{ 'dispCA', 'uncCA' \}. Indeed, by looking at the application to this uncertainty set of the $\mathrm{C}_{1}$ criteria a clear sensitivity analysis arises. Specifically, it is noticed that the pairs \{'dispXcp', 'uncXcp'\} and \{'dispCN', 'uncCN'\} can be set respectively to +1.0 and -1.0 while the parameters 'dispCA' and 'uncCA' can be focused respectively within $[-1.0,1.0]$ and $[-0.9,-0.38]$.
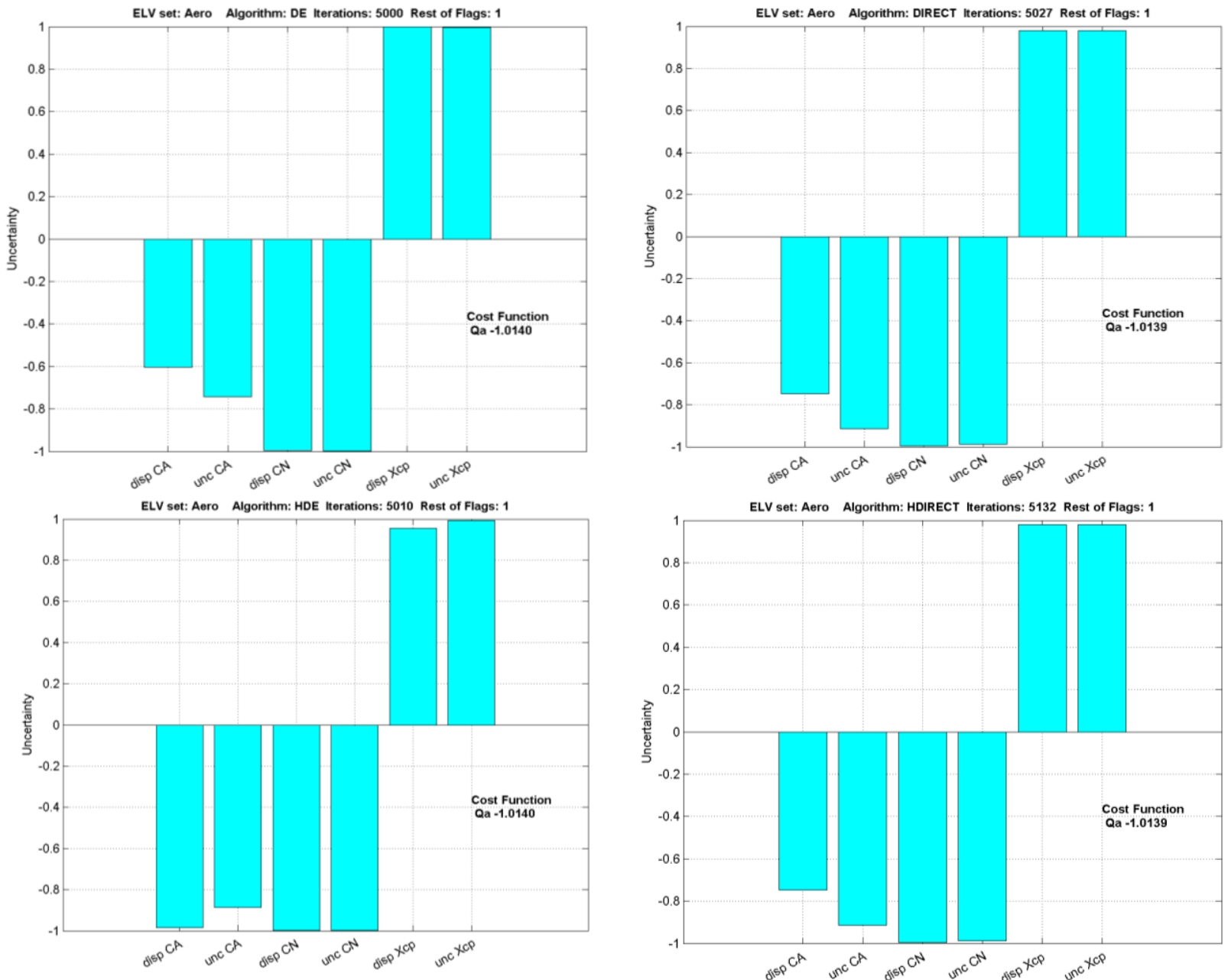

Figure 7 Aerodynamics set and 5K iterations, HDE \& HDIRECT for $\mathrm{C}_{1}$ : WCCs

7

American Institute of Aeronautics and Astronautics 
With respect to other cost functions, no instability case are found employing $C_{2}$ (based on roll rate) but several cases are found for the $\mathrm{C}_{3}$. In summary, for the Aerodynamic uncertainty set, the four algorithms found WCCs with as little as 1000 iterations using the cost functions $\mathrm{C}_{1}$ and $\mathrm{C}_{3}$. However, the WCCs where very different, for example for $\mathrm{C}_{1}$ the cases were found with all the other uncertainty flags set to +1.0 while for $\mathrm{C}_{3}$ it was possible to find WCC at any of the three values $\{-1.0,0.0,+1.0\}$. In the case of the $C_{2}$ cost function no algorithm was able to find a worst case condition even in the 5000 iterations case.

\section{B. MCI set}

The MIC set is composed by 10 parameters related to the launcher and propellant mass (' $\mathrm{dM}$ ' and 'dM Prop'), the stage and overall launcher $\mathrm{X}$ center of gravity coordinate ('dxCoG S', 'dxCoG'), the stage and overall launcher $\mathrm{X} / \mathrm{Y} / \mathrm{Z}$ moments of inertia ('dJx', 'dJy', 'dJz', 'JxS', 'JyS', and 'JzS')

For this set, the 1000 iterations case does not result in a constraint-violating WCC-see Figure 8. However, for the 5000 iterations case, the DE and HDE algorithms find WCCs using $C_{1}$ and $C_{3}$ respectively, see Figure 9. Notice that despite these later WCC being very dissimilar (Figure 9) they result in a very similar response for $Q \alpha$, see Figure 10.
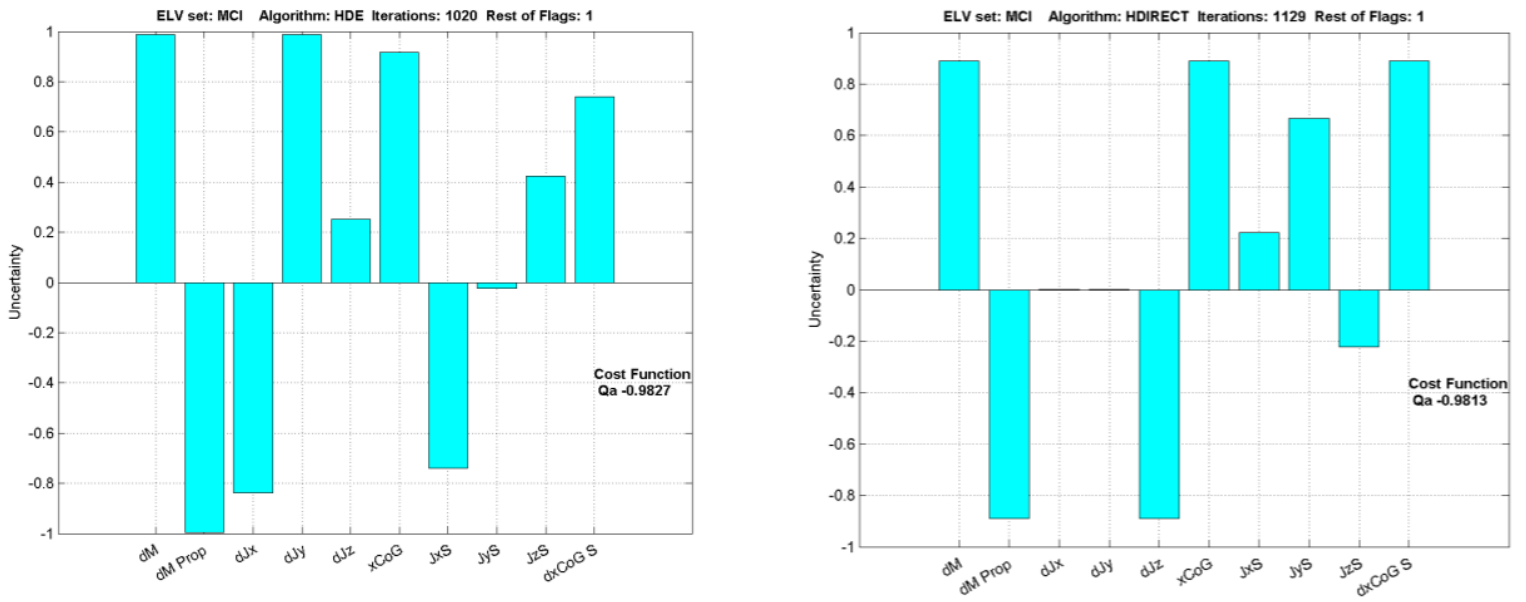

Figure 8 MCI set and 1k iterations, HDE \& HDIRECT for $\mathrm{C}_{1}$ : WCCs
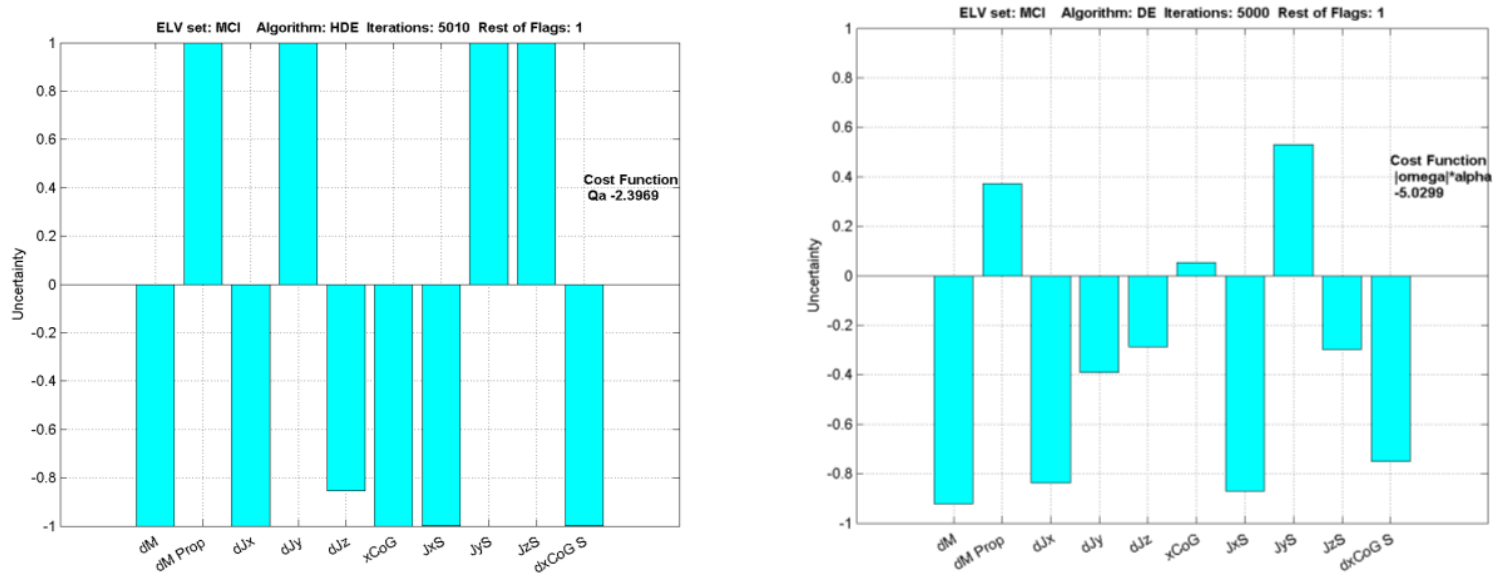

Figure 9 MCI set and 5k iterations, HDE for $\mathrm{C}_{1} \&$ DE for $\mathrm{C}_{3}$ : WCCs 

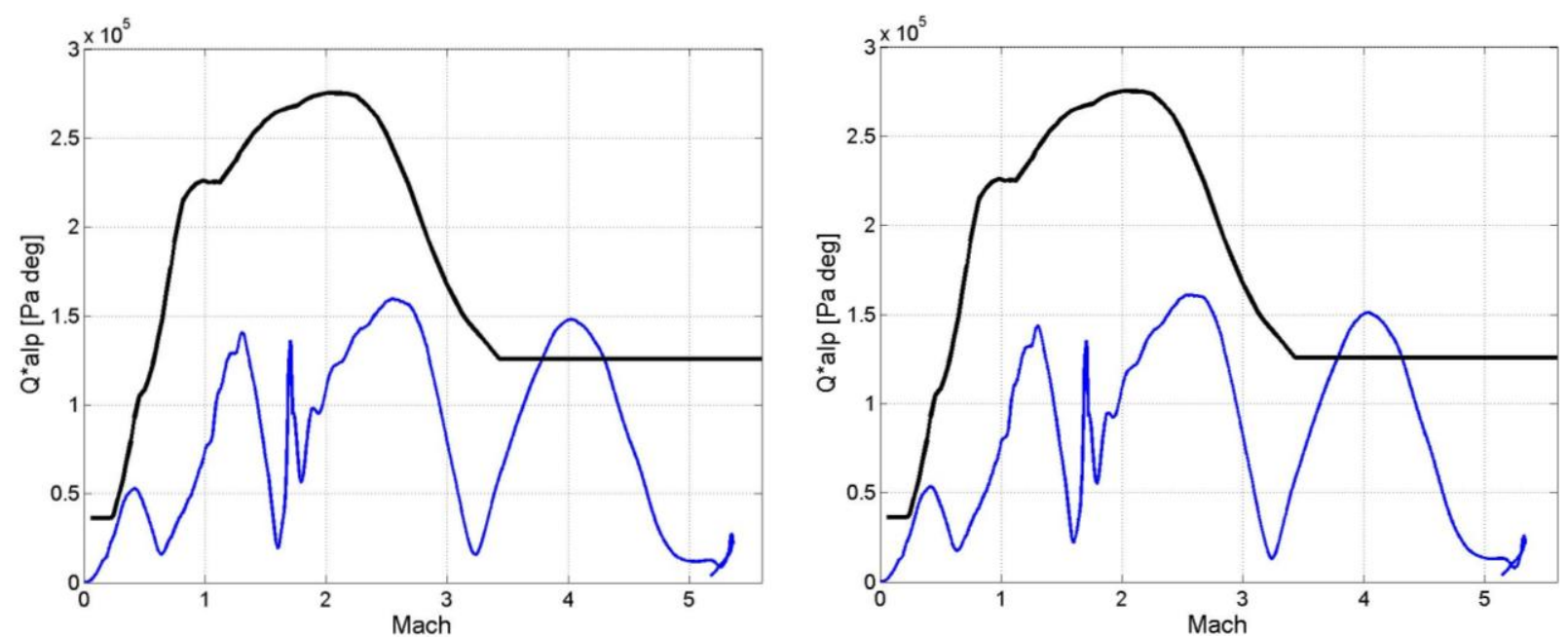

Figure $10 \mathrm{MCI}$ set and 5k iterations, $\mathrm{HDE} \mathrm{C}_{1} \& \mathrm{DE} \mathrm{C}_{3}$ : time-responses

\section{Global search set}

In order to better compare the WCC search algorithms to the traditional Monte Carlo of Section III, a global search with all the 95 uncertain parameters random is performed using the four algorithms. The results for the DE and HDE algorithms are better than expected, since they are able to find constraint-violating WCCs with as little as 1000 iterations (greatly surpassing the traditional MC which could not find one with 100,000 combinations!!!), see the function cost value in the headings of Figure 11. Further, note that most parameters have values well within the uncertainty range, clearly indicating that a vertex approach will not be able to detect them.
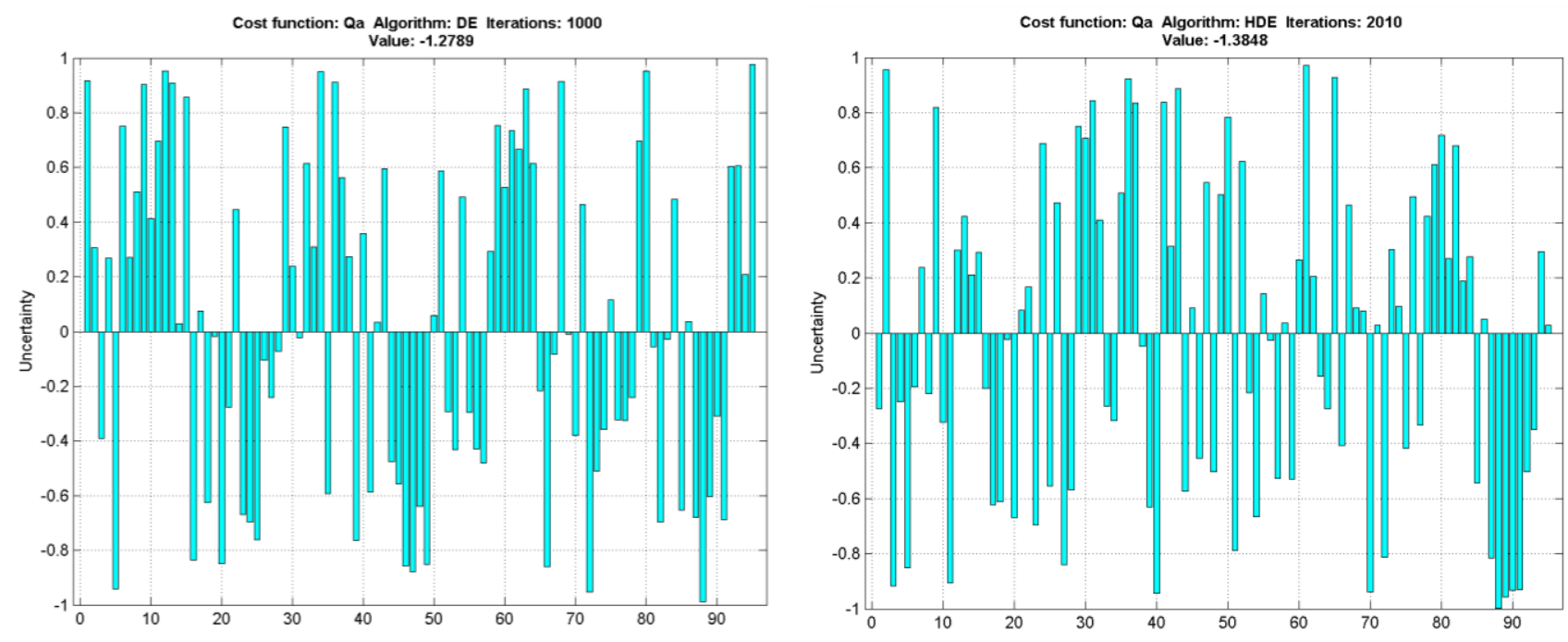

Figure 11 Global set and $1 \mathrm{~K}$ iterations, DE and HDE for $\mathrm{C}_{1}$ : WCCs

It is also interesting to see that the DIRECT and HDIRECT are not able to find any constraint-violating WCC although they get very close to the bound $\left(\left|\mathrm{C}_{1}\right|=0.9583\right)$ by perturbing only four of the parameters, see Figure 12 . The larger challenge for these two algorithms lies, as it was noted before, in the way they build their dividingrectangles which is based on the number of parameters and thus for large parameter spaces they may require large number of iterations and 'good' hybridization' rules to avoid local minima. 

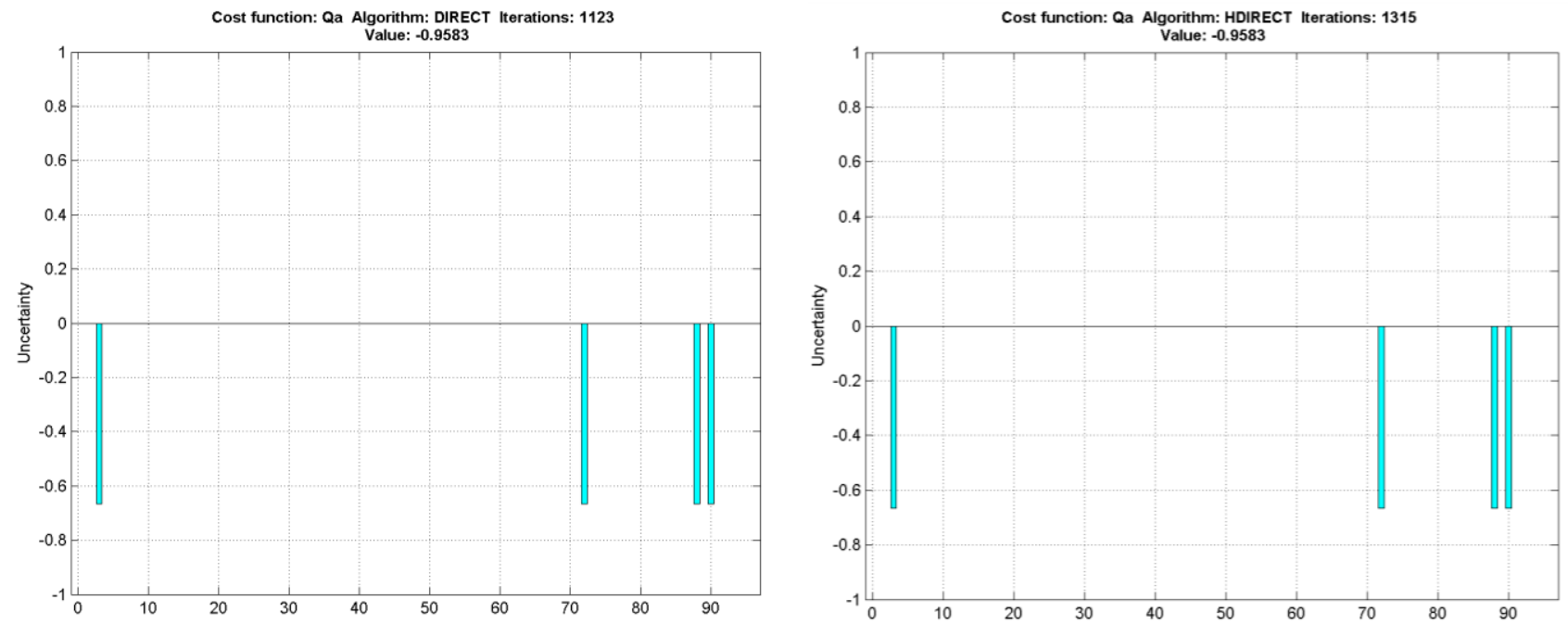

Figure 12 WCC found by DIRECT and HDIRECT algorithms for $\mathrm{C}_{1}$ using Global set

\section{VEGACONTROL versus VEGAMATH}

The presented worst-case combinations shown before were obtained applying the optimization-based algorithms to the VEGACONTROL simulator (see Section B), which is a simplified nonlinear simulator with respect to the official VEGAMATH developed to perform fast simulations.

A total of 58 WCCS were identified during the tests, see the left plot in Figure 13 for their Q $\alpha$ response in VEGACONTROL, which then were validated by ELV in VEGAMATH, see the 10 most critical (in terms of $\mathrm{C}_{*}$ violation) on the right plot of the figure. As it is seen, the results between both simulators map very well which serve to validate not only the fidelity of VEGACONTROL for this type of tests but also the use of optimization-based worst-case search algorithms to quickly detect WCCs.
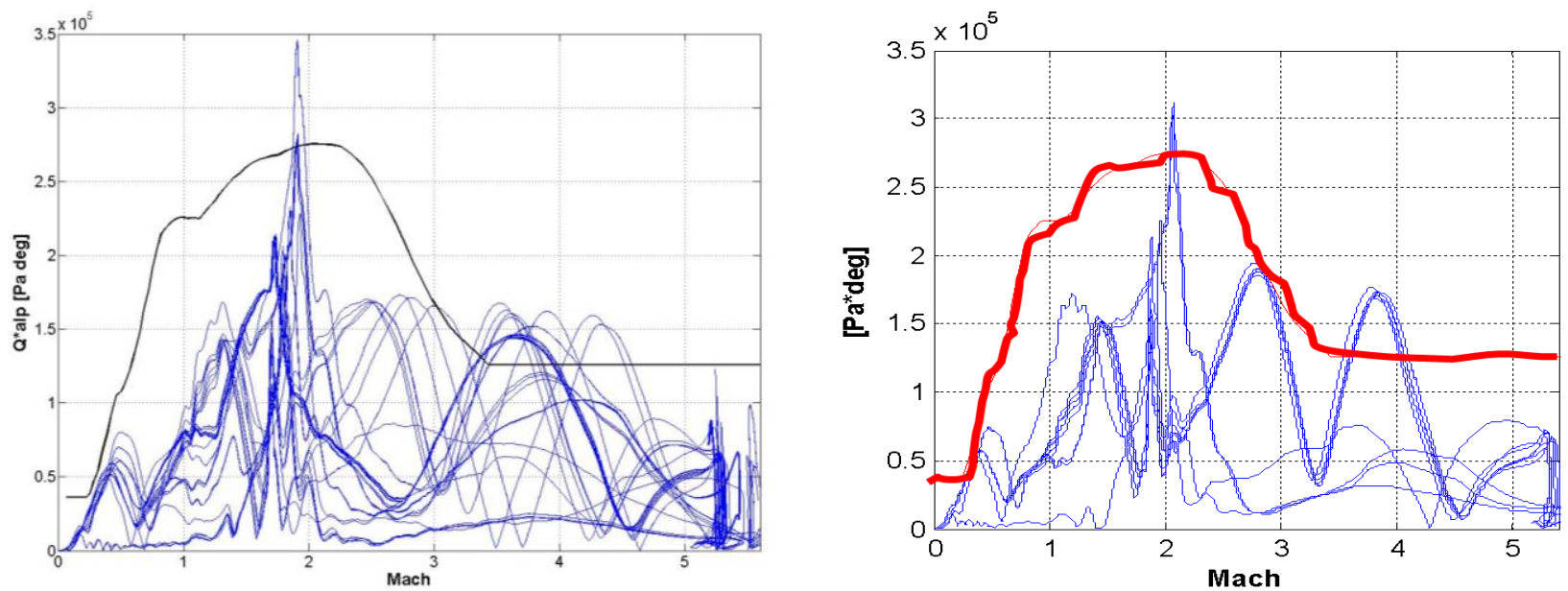

Figure 13 Time response of WCCs in VEGACONTROL (left) and VEGAMATH (right) for Q $\alpha$ 


\section{Conclusions}

It is well-known that traditional Monte Carlo campaigns, despite their use in industry and control certification, are not suitable for searching worst-case conditions or even to clear a parameter space. Due to this, a shift to perform the clearance using optimization-based algorithms has occurred in the past 15 years.

In the present study, four optimization algorithms were used: Differential Evolution (DE), Hybrid Differential Evolution (HDE), Dividing Rectangles (DIRECT) and Hybrid Dividing Rectangles (HDE). In order to help compare better the algorithms properties while performing a sensitivity analysis, the uncertain parameters were divided into several non-overlapped uncertainty subsets and then the optimization algorithms were applied to each using a set of cost-functions and different number of iterations.

From the results it is very clear that:

- Traditional MC, despite using 100,000 random combinations, was not useful to obtain worst-case conditions (WCC).

- The search algorithms are able to identify WCCs even with as little as 1000 iterations by using some minor engineering knowledge $-i$.e. the split of the parameter space into subsets and setting iteratively all the non-evaluated set parameters to vertex values $\{-1.0,0.0,+1.0\}$.

- For a global search (i.e. setting all the 95 uncertainty parameters as the optimization vector), it was found that the DE and HDE are the most suitable algorithms. This, of course, is only valid for the case of the VEGA launcher during P80 phase and further, it is noted that more appropriate hybridization rules might yield better results.

- In terms of the cost function, it was found that $\mathrm{C}_{3}$ and $\mathrm{C}_{1}$ are the best to detect WCCs (but even for $\mathrm{C}_{2}$ sometimes a WCC was identified).

In general, for all the algorithms, the larger the number of iterations used the better the result. Also, most of the WCC were found in the interior of the parameter space, which means that a pure vertex approach has less chance of identifying conditions (although engineering judgement could combine sets so as to get close to constrain-violating results).

Finally, the identified WCCs were validated in the official VEGA nonlinear simulator and yielded similar performance violations. These violations are acceptable in VEGA V\&V but the conclusion of the RFCS study has identified that the use of the presented optimization-based worst-case search algorithms and tools is a very relevant direction to further explore and incorporate to the launcher $\mathrm{V} \& \mathrm{~V}$ due to its fast capability of identifying WCCs but also the simplicity of use and the easiness of incorporating engineering knowledge to explore specific regions or parameters' combinations.

\section{References}

${ }^{1}$ Fielding et al., Advanced Techniques for Clearance of Flight Control Laws, Lecture Notes in Control and Information Science. Berlin: Springer-Verlag, 2002.

2 Jacklin, S.A., Schumann, J.M., Gupta, P.P., Richard, R., Guenther, K., and Soares, F., "Development of Advanced Verification and Validation Procedures and Tools for the Certification of Learning Systems in Aerospace Applications," Proceedings of Infotech aerospace Conference, Arlington, VA, Sept. 26-29, 2005.

${ }^{3}$ Belcastro, C. and Belcastro, C., "On the Validation of Safety Critical Aircraft Systems, Part II: An Overview of Analytic and Simulation Methods," AIAA Guidance, Navigation and Control Conference, AIAA-2003-5560, August 2003.

${ }^{4}$ A. Marcos, C. Roux, M. Rotunno, H. Joos, S. Bennani, L.F. Peñín, A. Caramagno, "The V\&V problematic for launchers: current practice and potential advantages on the application of modern techniques," ESA GNC 2011

${ }^{5}$ R. Storn and K. Price, "Differential evolution: a simple and efficient heuristic for global optimization over continuous space", Journal of Global Optimization, Vol.11, pp. 341-369, 1997.

${ }^{6}$ J. Lampinen and I. Zelinka, "Mechanical engineering design by differential evolution", David Corne, Marco Dorigo and Fred Glover (eds.) New Ideas in Optimization, McGraw Hill, London (UK), pp. 127-146, 1999.

${ }^{7}$ T. Rogalsky, R. W. Derksen and S. Kocabiyik, "Differential evolution in aerodynamic optimization", Canadian Aeronautics and Space Institute Journal, Vol.46, pp. 183-190, 2000.

${ }^{8}$ J. Yen, J. C. Liao, D. Randolph, and B. Lee, "A hybrid approach to modeling metabolic systems using genetic algorithm and simplex method", Proc. of the 11th IEEE Conference on Artificial Intelligence for Applications, pp. 277-283, Los Angeles, CA, Feburary 1995. 
${ }^{9}$ F.G. Lobo and D.E. Goldberg, Decision making in a hybrid genetic algorithm, IlliGAL Report No. 96009, September 1996.

${ }^{10}$ T. Rogalsky and R.W. Derksen, "Hybridization of differential evolution for aerodynamic design", Proc. of the 8th Annual Conference of the Computational Fluid Dynamics Society of Canada, pp. 729-736, 2000.

${ }^{11}$ D. E. Finkel and C. T. Kelley, Convergence Analysis of the DIRECT Algorithm, N. C. State University Center for Research in Scientific Computation Tech Report number CRSC-TR04-28, 2004.

${ }^{12}$ H., Zhu, and D. B., Bogy, DIRECT Algorithm and its application to slider air-bearing surface optimization,IEEE Transactions on Magnetics, Vol. 5, 2002.

${ }^{13}$ Menon, P.P., Prempain, E., Postlethwaite, I., Bates, D.G., "Nonlinear Worst-Case Analysis of an LPV Controller for Approach-Phase of a Re-Entry Vehicle," AIAA GNC 2009

${ }^{14}$ Marcos, A., Bennani, S., "LPV Modeling, Analysis and Design in Space Systems: Rationale, Objectives and Limitations," AIAA GNC 2009. 